\title{
Clinical efficacy of resveratrol as an adjuvant with meloxican in the treatment of knee osteoarthritis patients: A double-blind, randomised, placebo-controlled trial
}

\author{
Bushra Hassan Marouf 1 , Saad Abdulrahman Hussain ${ }^{2 *}$, Ziyad Serdar Ali ${ }^{3}$, Runj Simko Ahmmad $^{3}$ \\ ${ }^{1}$ Department of Pharmacology and Toxicology, College of Pharmacy, University of Sulaimani, Kurdistan Region, ${ }^{2}$ Faculy of \\ Pharmacy, Al-Rafidain University College, Baghdad, Iraq, ${ }^{3}$ Department of Rheumatology and Orthopedics, Shar Teaching \\ Hospital, Sulaimani, Kurdistan Region, Iraq
}

\begin{abstract}
The present study aimed to evaluate the effect of the adjuvant use of resveratrol with meloxicam on the clinical scores of knee OA patients. This was a double-blind placebo-controlled randomised trial involving 100 patients with knee osteoarthritis performed at the Shar Teaching Hospital, Sulaimani General Hospital and Specialised Rheumatology Center, Sulaimani City from December 2016 to September 2017. The efficacy of the treatment was evaluated by measuring the changes from baseline in the KOOS score, WOMAC index, and VAS-100 score after 90 days of treatment. Resveratrol significantly improves the knee OA pain and associated symptoms compared with placebo, and both clinical scores were found to be eligible for following treatment outcomes. In conclusion, resveratrol, when used in combination with meloxicam, improves pain and symptom scores in patients with mild-to-moderate knee OA compared with placebo. The intervention with a dietary supplement may significantly impact the pain and overall quality of life in patients with knee OA.
\end{abstract}

Keywords: Resveratrol/effects. Meloxicam/effects. Knee OA. KOOS. WOMAC.

\section{INTRODUCTION}

Knee osteoarthritis (OA) is a slowly progressive chronic degenerative and disabling disease of the articulating knee joints, which mostly arises after hyaline cartilage damage (Loeser et al., 2012). Although it is considered the most common musculoskeletal disorder mainly affecting people over 65 years old (Mobasheri, Batt, 2016), it also affects active younger patients, as a result of physical trauma or due to the long-term participation in highly demanding sports (Buckwalter, Martin, 2004). As a result of continuous progressive inflammatory changes that predispose to many painful symptoms, knee OA affects many aspects of the patient's life, including the ability to perform normal daily functions, sitting, walking and climbing stairs (Kraus et al., 2015), which may result in a poor quality of life (Johnson, Hunter, 2014). Moreover, knee OA is highly

\footnotetext{
*Correspondence: S. A. Hussain. Faculy of Pharmacy, Al-Rafidain University College Baghdad, Iraq. Phone: +9647901712624. E-mail: saad.hussain@coalrafidain.edu.iq
}

associated with morbidity in the community (Hayami, 2008). In spite of advances in the elaboration of cellular and molecular mechanisms of OA pathogenesis and the identification of more sensitive and reliable biomarkers as diagnostic tools (Mobasheri et al., 2017), there is no optimal therapy that slows cartilage breakdown or reverses the progression of cartilage degradation and other tissue damage. In addition to non-pharmacological approaches to treatment, many drugs are used in the pharmacological treatment of OA; non-steroidal anti-inflammatory agents (NSAIDs) are the most widely used for their analgesic and anti-inflammatory activities, which effectively reduce the symptoms of OA (Nelson et al., 2014). However, the long-term use of these drugs was correlated with many important adverse events, including an increased risk of gastrointestinal bleeding, cardiovascular disorders and an increased bleeding tendency due to platelet activation (Zhang et al., 2007; Varas-Lorenzo et al., 2013). Currently, there is a growing interest in developing therapeutic strategies to decrease the progressive degeneration of joint tissues with fewer side effects. In this regard, numerous studies have recently focused on the potential effects of 
natural compounds, including nutraceuticals, to halt the progression of OA and reduce any associated symptoms (Hussain et al., 2009; Moura et al., 2016). Resveratrol is a natural polyphenol extracted from Polygonum cuspidatum, which shows pleiotropic properties that suggest it may be applied for many therapeutic uses, including the treatment of cancer, cardiovascular diseases, and metabolic disorders (Neves, Nunes, Reis, 2016). Meanwhile, many preclinical studies have revealed the anti-inflammatory properties of resveratrol that might enable the degenerative articular damage to be ameliorated in vitro and in experimental animals (Jiang et al., 2017; Ma et al., 2015). Additionally, resveratrol inhibits the destruction of the cartilage matrix by protecting matrix proteins, proteoglycans, collagen type II and aggrecans, against the effects of the matrix metalloproteinases (MMPs) or inflammatory stimuli (i.e., iNOS, COX2) (Nguyen et al., 2017). Pre-clinical findings support the safety of resveratrol, and toxicity studies on different animal species did not reveal any serious adverse effects (Williams et al., 2009; Johnson et al., 2011). Based on previous data, resveratrol did not produce any apparent adverse effects up to a dose of $1.0 \mathrm{~g}$ in the short-term. However, when patients administered $2.5 \mathrm{~g} /$ day or more, diarrhoea, vomiting, nausea and evidence of liver dysfunction were reported in patients with nonalcoholic fatty liver disease (Brown et al., 2010; La Porte et al., 2010). Interestingly, no serious side effects were reported in long-term clinical trials using low doses of resveratrol (Tomé-Carneiro et al., 2013), and resveratrol was found to be safe and reasonably well-tolerated up to $5.0 \mathrm{~g} /$ day, either as a single dose or as part of a multipleday dosing regimen (Patel et al., 2011). In spite of the presence of a tremendous amount of in vitro and in vivo data on the chondroprotective and regenerative capacity of resveratrol, no clinical trials exist to date that have determined the effectiveness of resveratrol in the treatment of knee OA. The present study was designed to evaluate the effect of adjuvant use of resveratrol with meloxicam on the clinical scores of knee OA patients.

\section{MATERIAL AND METHODS}

\section{Study design}

A prospective double-blind placebo-controlled randomised multi-centre trial was performed to evaluate the efficacy of adjuvant use of resveratrol (Res) with meloxicam (Mlx) against placebo (starch powder) on improving the symptoms of knee OA. The present study was conducted over a period of 10 months; the first patient was included in December 2016 and the last patient completed follow-up in September 2017. The research was conducted at Shar Teaching Hospital, Sulaimani General Hospital and the Specialised Rheumatology Center, Sulaimani City. The research protocol was approved by the Research Ethics Committee of the College of Medicine, University of Sulaimani (No. 42 in 21/11/2016) in accordance with the Declaration of Helsinki and its amendments, as well as the Guidelines for Good Clinical Practices issued by the Committee of Propriety Medicinal Products of the European Union. All participants were asked to sign a written informed consent voluntarily before participation in the study. Patient safety was closely monitored by a board of specialists by conducting regular meetings during the study.

\section{Patient inclusion and randomisation}

One hundred and fourteen patients were screened for eligibility. Based on the criteria for OA diagnosis (American College of Rheumatology; ACR), and the methods found in previously published studies, a hundred eligible patients of both sexes (age range 45-75 years) with clinical evidence of knee OA were diagnosed by a senior rheumatologist and orthopaedic clinician according to the American College of Rheumatology criteria (Altman et al., 1986); the results of radiographic diagnosis were of mild-to-moderate knee OA (grade I-III) according to the Kellgren-Lawrence classification (Kellgren, Lawrence, 1957), defined by the presence of typical knee symptoms (pain, stiffness, disability) and radiographic markers of OA. Other inclusion criteria were knee complaints surpassing the threshold indicated by at least two of the following questionnaires: maximal 60 points out of 96 points on the Western Ontario and McMaster Universities Osteoarthritis Index (WOMAC), maximal 40 points on the Knee injury and Osteoarthritis Outcome Score (KOOS), and minimal $40 \mathrm{~mm}$ for the 100-mm Visual Analogue Scale (VAS) for pain. Patients were excluded if they had an established diagnosis of a bleeding disorder; currently used antioxidant-containing drugs or dietary supplements that contain polyphenols, anticoagulants, or antiplatelet therapy; or presence of an allergy to any of the ingredients used in the present study. The use of NSAIDs, other than that indicated during the trial period (meloxicam), was not allowed. The participants were advised not to use any other analgesics or anti-inflammatory agents. The participants were randomised (3:2 ratio) by independent outpatient units. A simple randomisation technique was followed, in which the predetermined sample size for treatment group $(n=60)$ and placebo $(n=40)$ were shuffled in opaque envelopes containing an identifier for treatment group 
and an identifier for placebo. The order of the shuffled envelopes determined the allocation of participants to one of the treatments. The process was relatively simple to organise, preserves the predetermined design parameters, and can be readily extended to situations of long recruitment duration. The control group initiated treatment with $15 \mathrm{mg}$ meloxicam (Standardised 98\% pure; Boehringer Ingelheim, Germany) and placebo formula specially prepared for the study $(\mathrm{Mlx}+$ placebo) orally once per day for 90 days; the resveratrol group received $15 \mathrm{mg}$ meloxicam with $500 \mathrm{mg}$ resveratrol (Sigma-Aldrich, UK) orally once per day for 90 days $(\mathrm{Mlx}+\mathrm{Res})$. Based on the literature review, most of the investigations on bioactive polyphenols need no less than 90 days for the treatment period to achieve a satisfactory response such as analgesic and/or anti-inflammatory effects. The placebo was formulated as hard gelatine capsules with an identical colour, form and size as the resveratrol capsules. All of those involved in the trial (investigators, participants, staff collecting data, and statistician) were blinded to the randomisation. The patients using anti-inflammatory drugs before inclusion were held in a 2 week washout period. During this period, oral or local administration was gradually withdrawn within a week after enrolment and then the patients did not receive any drug for the following week; after this, each patient was assigned to one of the treatment groups of the trial. Recruited patients were asked to carry on with their regular daily activities that they had been undertaking before inclusion and were also advised to continue the same until the end of the trial period. Additionally, patients were asked to return the empty containers of trial drugs at every follow-up visit in order to check their compliance with the protocol of drug use. The patients were free to stop participating in the study at any time without prior permission of the investigator or any reason. Furthermore, the investigator was able to remove any patient from the study who developed adverse effects or showed non-compliance with the treatment protocol.

\section{Follow-up assessment}

The included patients attended the assigned outpatient clinic for follow-up visits on day 0 (First visit), day 30 (Second visit), day 60 (Third visit), and day 90 (Last visit). During each follow-up visit, general and systemic physical examinations were performed. The evaluation of knee OA symptoms was performed utilising the total KOOS score (Salavati et al., 2011), total WOMAC index (Bellamy et al., 1988) and visual analogue score for pain
(VAS-100) (Haq, Davatchi, 2011) to assess pain as a primary outcome. Global assessment of disease severity score was assessed by both the investigators and subjects on every follow-up visit to determine the efficacy of the treatment. As a primary outcome, the efficacy of the treatment was evaluated by measuring the change from baseline in the KOOS score, WOMAC index, and the VAS-100 score; these were measured at baseline, and at 30, 60 and 90 days of the out-patient visits. The secondary outcomes were measurement of the changes in KOOS subscale scores (symptom, pain, daily activity, sport and quality of life) of knee OA severity, changes in WOMAC subscale scores (stiffness, pain and daily functions), adverse drug reactions, and compliance with treatment protocols. Adherence to the medication protocol was monitored through behavioural and telephone-based interventions on regular monthly follow-up bases.

\section{Statistical analysis}

The analysis of data was performed using GraphPad Prism 5.0.1 program (GraphPad Software Inc., CA, USA). The baseline demographic data and patient characteristics were compared between the treatment groups using the Chi-square test for categorical and unpaired $t$-test to compare between the means of different groups at baseline. Continuous variables were analysed using the paired $t$-test and the two-way ANOVA confirmed by Bonferroni's post hoc test. Spearman's correlation and ROC analysis were utilised to compare the outcomes of questionnaires. A $p$ value $<0.05$ was considered to indicate statistical significance.

\section{RESULTS}

A total of 114 patients were screened for eligibility; of these, 100 met the inclusion criteria, and were randomised and assigned to the trial. Only 82 patients completed the follow-up: 10 from the Mlx+Res group, and 8 from the $\mathrm{Mlx}+$ placebo group did not complete the follow-up visits for various reasons (Figure 1).

\section{Baseline characteristics}

The baseline comparative demographic data between the patients with knee OA in the Mlx+placebo and $\mathrm{Mlx}+$ Res groups are shown in Table I. There were no significant differences in age, sex, body weight, body mass index, and disease grade or disease duration between the two groups. Sex was not a potential variable in the present study based on the absence of any differences in 


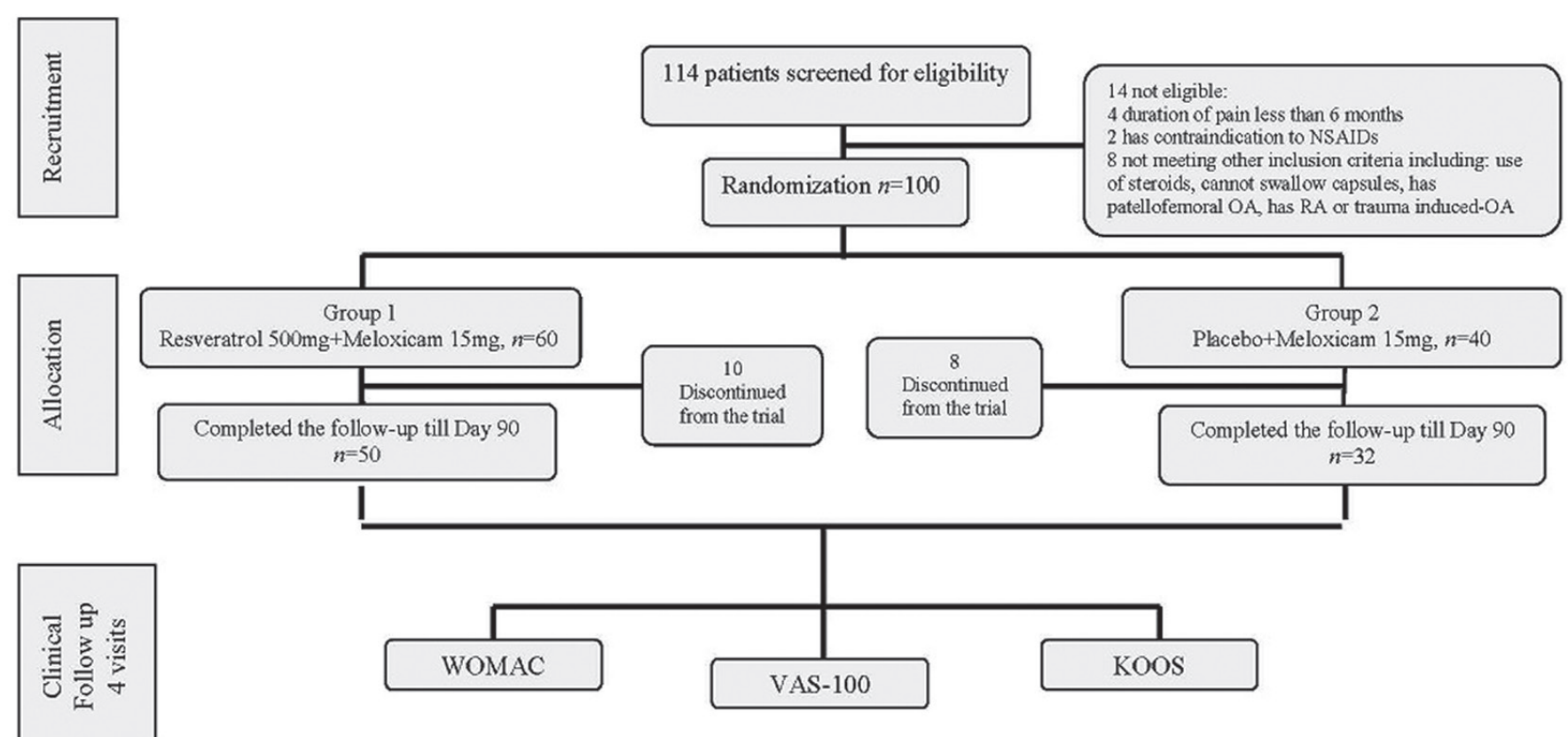

FIGURE 1 - Flow chart of patient inclusion and follow-up. $n$ : number of patients; OA: osteoarthritis; RA: rheumatoid arthritis; WOMAC: Western Ontario and McMaster Universities Arthritis Index; VAS: Visual Analogue Score; KOOS: Knee injury and Osteoarthritis Outcome Score.

TABLE I - Demographic data and baseline characteristics of the Knee OA patients treated with Meloxicam+Resveratrol (Mlx+Res) or Meloxicam+placebo (Mlx+placebo)

\begin{tabular}{|c|c|c|c|}
\hline Parameters & Res+Mlx, $n=50$ & Mlx + placebo, $n=32$ & $P$ value \\
\hline \multicolumn{4}{|l|}{ Gendern (\%) } \\
\hline Male & $13(26)$ & $6(9.4)$ & 0.03 \\
\hline Female & $37(74)$ & $14(90.6)$ & 0.06 \\
\hline Age (year) & $58.2 \pm 9.1$ & $57.6 \pm 8.2$ & 0.77 \\
\hline Body weight (kg) & $80.5 \pm 16.1$ & $82.4 \pm 11.0$ & 0.55 \\
\hline BMI $\left(\mathrm{kg} / \mathrm{m}^{2}\right)$ & $30.7 \pm 5.6$ & $32.1 \pm 4.6$ & 0.1 \\
\hline Disease duration (year) & $3.5 \pm 3.2$ & $3.9 \pm 2.9$ & 0.45 \\
\hline \multicolumn{4}{|l|}{ Disease grade $n(\%)$} \\
\hline Grade I & $7(14)$ & $3(9.4)$ & 0.12 \\
\hline Grade II & $25(50)$ & $15(47)$ & \\
\hline Grade III & $18(36)$ & $14(43.6)$ & \\
\hline Baseline KOOS & $33.6 \pm 9.2$ & $33.5 \pm 10.5$ & 0.9 \\
\hline Baseline WOMAC & $59.6 \pm 13.7$ & $59.3 \pm 7.4$ & 0.2 \\
\hline Baseline VAS-100 (mm) & $81.0 \pm 11.6$ & $85.3 \pm 9.2$ & 0.08 \\
\hline \multicolumn{4}{|l|}{ Associated diseases $n(\%)$} \\
\hline Hypertension & $20(40.0)$ & $18(56.0)$ & 0.10 \\
\hline Diabetes mellitus & $8(16.0)$ & $3(9.4)$ & 0.12 \\
\hline Smoking habits & $3(6.0)$ & $1(3.1)$ & 0.11 \\
\hline
\end{tabular}

Values are presented as percent or mean \pm S.D; $n$ : number of patients; Res: Resveratrol; Mlx: Meloxicam. Chi-square and unpaired $t$-test were utilised to predict significance at $P<0.05$. 
treatment response between males and females. Moreover, the baseline total KOOS, total WOMAC and VAS-100 scores were also not significantly different between the two groups.

\section{The clinical outcome}

Table II shows that adjuvant use of resveratrol with meloxicam significantly improves total KOOS score after 30 days compared with both baseline values and that of the $\mathrm{Mlx}+$ placebo group within the same period $(P<0.05)$. Although the effect of resveratrol continues to improve KOOS score after 60 and 90 days, these values are not significantly different $(P>0.05)$. The post-intervention KOOS score in the Mlx+placebo group was not significantly different for all of the time ranges. Moreover, the post-intervention WOMAC score in Mlx+Res was significantly improved at all of the time ranges compared with both baseline values and those of the Mlx + placebo group $(P<0.05)$. Meanwhile, the post-intervention WOMAC score was not significantly changed after 90 days in this group. Additionally, Table
II indicates that the post-intervention VAS-100 score was significantly improved in both groups compared to baseline; however, the degree of changes in Mlx+Res group was significantly higher compared to those reported in $\mathrm{Mlx}+$ placebo group. In Table III, the post intervention KOOS subscale score in Mlx+Res group was significantly improved in all KOOS areas compared with both baseline values and that reported in the $\mathrm{Mlx}+$ placebo group, which show non-significant changes in subscale score for the entire treatment period. Similarly, Table IV indicates that the post-intervention WOMAC subscale score in Mlx +Res group was significantly improved in all WOMAC subscale areas compared with baseline values and the corresponding results of $\mathrm{Mlx}+$ placebo group, where all of the subscale values were not significantly different during the treatment period. The validity of KOOS at different levels in the evaluation of treatment outcome in both groups compared with WOMAC was evaluated using Spearman's correlation, where a significant negative correlation was detected in the Mlx+placebo group at most of the values greater than $1(\mathrm{r}=-0.6, P=0.001)$ (Figure 2). The influence of

TABLE II - Effect of resveratrol (Res) as an adjuvant with meloxicam (Mlx) on the Knee Injury and Osteoarthritis Outcome Score (KOOS), Western Ontario and McMaster Universities Arthritis (WOMAC) index, and Visual Analogue Score (VAS-100) in patients with mild-to-moderate knee OA

\begin{tabular}{lcccccccc}
\hline \multirow{2}{*}{ Score } & \multicolumn{4}{c}{ Mlx+Res, $\boldsymbol{n}=\mathbf{5 0}$} & \multicolumn{4}{c}{ Mlx+placebo, $\boldsymbol{n}=\mathbf{3 2}$} \\
\cline { 2 - 8 } & Baseline & $\mathbf{3 0}$ days & $\mathbf{6 0}$ days & $\mathbf{9 0}$ days & Baseline & $\mathbf{3 0}$ days & $\mathbf{6 0}$ days & $\mathbf{9 0}$ days \\
\hline KOOS & $33.6 \pm 9.2^{\mathrm{a}}$ & $81.7 \pm 12.9^{* \mathrm{~b}}$ & $87.8 \pm 10.2^{* \mathrm{~b}}$ & $89.3 \pm 9.5^{* \mathrm{~b}}$ & $33.5 \pm 10.5^{\mathrm{a}}$ & $38.9 \pm 15.1^{\mathrm{a}}$ & $37.2 \pm 14.3^{\mathrm{a}}$ & $38.3 \pm 14.8^{\mathrm{a}}$ \\
WOMAC & $59.6 \pm 13.7^{\mathrm{a}}$ & $37.2 \pm 10.5^{* \mathrm{~b}}$ & $24.4 \pm 8.4^{* \mathrm{c}}$ & $18.0 \pm 5.5^{* \mathrm{c}}$ & $59.3 \pm 7.4^{\mathrm{a}}$ & $54.2 \pm 11.1^{\mathrm{a}}$ & $52.8^{\mathrm{a}} \pm 12.1^{\mathrm{a}}$ & $50.1 \pm 14.9^{\mathrm{a}}$ \\
VAS-100 & $81.0 \pm 11.6^{\mathrm{a}}$ & $29.4 \pm 11.5^{* \mathrm{~b}}$ & $22.3 \pm 12.5^{* \mathrm{~b}}$ & $18.6 \pm 10.8^{* \mathrm{~b}}$ & $85.3 \pm 9.2^{\mathrm{a}}$ & $72.7 \pm 17.4^{* \mathrm{~b}}$ & $70.9 \pm 14.9^{* \mathrm{~b}}$ & $65.7 \pm 16.8^{* \mathrm{~b}}$ \\
\hline
\end{tabular}

Values are presented as mean \pm S.D; $n$ : number of patients; * significantly different compared with baseline values within the same group (paired $t$-test); values with different superscripts (a,b,c) are significantly different among different times and different groups (unpaired $t$-test and ANOVA; $P<0.05$ ).

TABLE III - Effect of resveratrol (Res) as adjuvant with meloxicam (Mlx) on the different areas of Knee injury and Osteoarthritis Outcome Score (KOOS) in patients with mild-to-moderate knee OA

\begin{tabular}{lcccccccc}
\hline \multirow{2}{*}{ KOOS area } & \multicolumn{4}{c}{ Mlx+Res, $\boldsymbol{n}=\mathbf{5 0}$} \\
\cline { 2 - 9 } & Baseline & $\mathbf{3 0}$ days & $\mathbf{6 0}$ days & $\mathbf{9 0}$ days & Baseline & $\mathbf{3 0}$ days & $\mathbf{6 0}$ days & $\mathbf{9 0}$ days \\
\hline Symptom & $49.0 \pm 20.2^{\mathrm{a}}$ & $84.8 \pm 12.6^{* \mathrm{~b}}$ & $89.7 \pm 11.8^{* \mathrm{~b}}$ & $92.1 \pm 9.6^{* \mathrm{~b}}$ & $38.3 \pm 17.0^{\mathrm{a}}$ & $44.1 \pm 19.6^{\mathrm{a}}$ & $43.8^{*} \pm 19.9^{\mathrm{a}}$ & $41.6 \pm 22.0^{\mathrm{a}}$ \\
Pain & $32.9 \pm 14.1^{\mathrm{a}}$ & $80.2 \pm 15.1^{* \mathrm{~b}}$ & $87.9 \pm 13.0^{* \mathrm{c}}$ & $90.3 \pm 12.9^{* \mathrm{c}}$ & $30.3 \pm 12.9^{\mathrm{a}}$ & $39.1 \pm 18.8^{\mathrm{a}}$ & $34.7 \pm 17.6^{\mathrm{a}}$ & $35.5 \pm 19.0^{\mathrm{a}}$ \\
Daily living & $38.4 \pm 14.8^{\mathrm{a}}$ & $83.7 \pm 16.4^{* \mathrm{~b}}$ & $90.3 \pm 10.3^{* \mathrm{~b}}$ & $91.6 \pm 7.8^{* \mathrm{~b}}$ & $35.1 \pm 12.2^{\mathrm{a}}$ & $39.6 \pm 16.2^{\mathrm{a}}$ & $39.7 \pm 15.5^{\mathrm{a}}$ & $40.9 \pm 16.1^{\mathrm{a}}$ \\
Sport & $20.8 \pm 15.7^{\mathrm{a}}$ & $63.0 \pm 17.5^{* \mathrm{~b}}$ & $75.2 \pm 14.9^{* \mathrm{c}}$ & $74.6 \pm 15.5^{* \mathrm{c}}$ & $24.5 \pm 8.8^{\mathrm{a}}$ & $30.2 \pm 13.4^{\mathrm{a}}$ & $23.8^{*} 14.0^{\mathrm{a}}$ & $28.6 \pm 9.8^{\mathrm{a}}$ \\
QoL & $35.3 \pm 10.6^{\mathrm{a}}$ & $77.1 \pm 18.5^{* \mathrm{~b}}$ & $85.6 \pm 11.1^{* \mathrm{c}}$ & $88.3 \pm 13.9^{* \mathrm{c}}$ & $29.9 \pm 11.2^{\mathrm{a}}$ & $36.7 \pm 14.2^{\mathrm{a}}$ & $36.3 \pm 14.3^{\mathrm{a}}$ & $39.1 \pm 10.9^{\mathrm{a}}$ \\
\hline
\end{tabular}

Values are presented as mean \pm S.D; $n$ : number of patients; Res: Resveratrol; Mlx: Meloxicam; QoL: Quality of life; * significantly different compared with baseline values within the same group (paired $t$-test); values with different superscripts $(\mathrm{a}, \mathrm{b}, \mathrm{c})$ are significantly different among different times and different groups (unpaired $t$-test and ANOVA; $P<0.05$ ). 
TABLE IV - Effect of resveratrol (Res) as an adjuvant with meloxicam (Mlx) on the different areas of Western Ontario and McMaster Universities Osteoarthritis Index (WOMAC) in patients with mild-to-moderate knee OA.

\begin{tabular}{lcccccccc}
\hline WOMAC & \multicolumn{5}{c}{ Mlx + Res, $\boldsymbol{n}=\mathbf{5 0}$} \\
area & Baseline & $\mathbf{3 0}$ days & $\mathbf{6 0}$ days & $\mathbf{9 0}$ days & Baseline & $\mathbf{3 0}$ days & $\mathbf{6 0}$ days & $\mathbf{9 0}$ days \\
\hline Stiffness & $4.3 \pm 2.2^{\mathrm{a}}$ & $1.1 \pm 1.3^{* \mathrm{~b}}$ & $0.8 \pm 1.3^{* \mathrm{~b}}$ & $0.7 \pm 1.2^{* \mathrm{~b}}$ & $5.8 \pm 1.4^{\mathrm{a}}$ & $5.3 \pm 1.8^{* \mathrm{~b}}$ & $5.5 \pm 1.9^{* \mathrm{~b}}$ & $4.6 \pm 2.3^{\mathrm{b}}$ \\
Pain & $13.4 \pm 3.3^{\mathrm{a}}$ & $3.6 \pm 3.1^{* \mathrm{~b}}$ & $2.1 \pm 2.6^{* \mathrm{~b}}$ & $1.8 \pm 2.5^{* \mathrm{~b}}$ & $13.8^{\mathrm{b}} \pm 2.4^{\mathrm{a}}$ & $12.1 \pm 4.0^{\mathrm{a}}$ & $12.2 \pm 4.0^{\mathrm{a}}$ & $11.9 \pm 4.4^{\mathrm{a}}$ \\
Function & $41.8 \pm 9.3^{\mathrm{a}}$ & $11.3 \pm 11.1^{* \mathrm{~b}}$ & $7.2 \pm 7.4^{* \mathrm{~b}}$ & $5.9 \pm 5.4^{* \mathrm{~b}}$ & $44.1 \pm 8.3^{\mathrm{a}}$ & $41.1 \pm 16.2^{\mathrm{a}}$ & $39.6 \pm 11.6^{\mathrm{a}}$ & $37.1 \pm 12.1^{\mathrm{a}}$ \\
\hline
\end{tabular}

Values are presented as mean \pm S.D; $n$ : number of patients; Res: Resveratrol; Mlx: Meloxicam; ${ }^{*}$ significantly different compared with baseline values within the same group (paired $t$-test); values with different superscripts $(\mathrm{a}, \mathrm{b})$ are significantly different among different times and different groups (unpaired $t$-test and ANOVA; $P<0.05$ ).
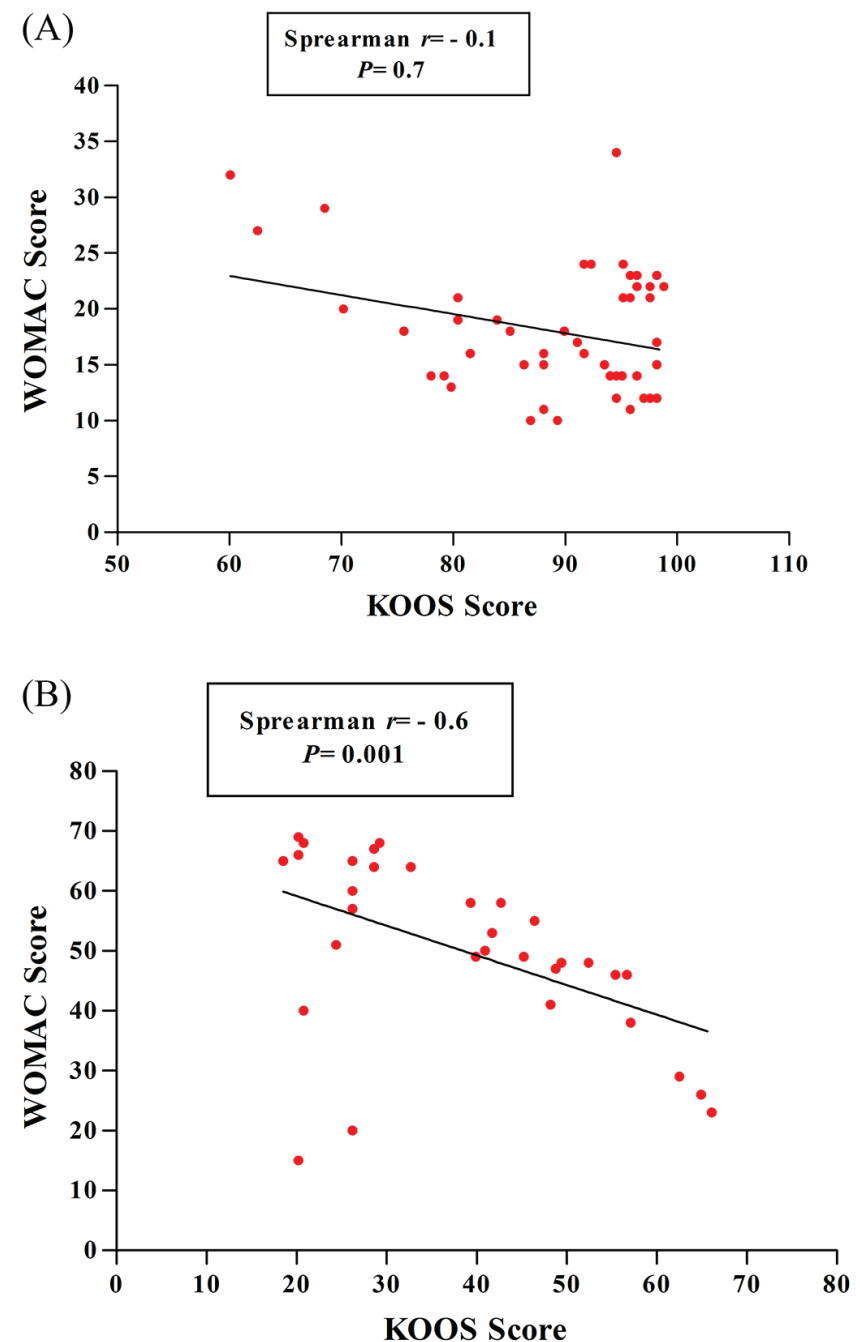

FIGURE 2 - Spearman's correlation between KOOS and WOMAC scores in both treatment groups after 90 days. $r$ : Spearman's correlation coefficient; A: Mlx+Res group; B: $\mathrm{Mlx}+$ placebo group.

both treatment approaches on the pain score was quite evident; Figure 3 shows the correlation between KOOS score and VAS-100 score, where a high negative and
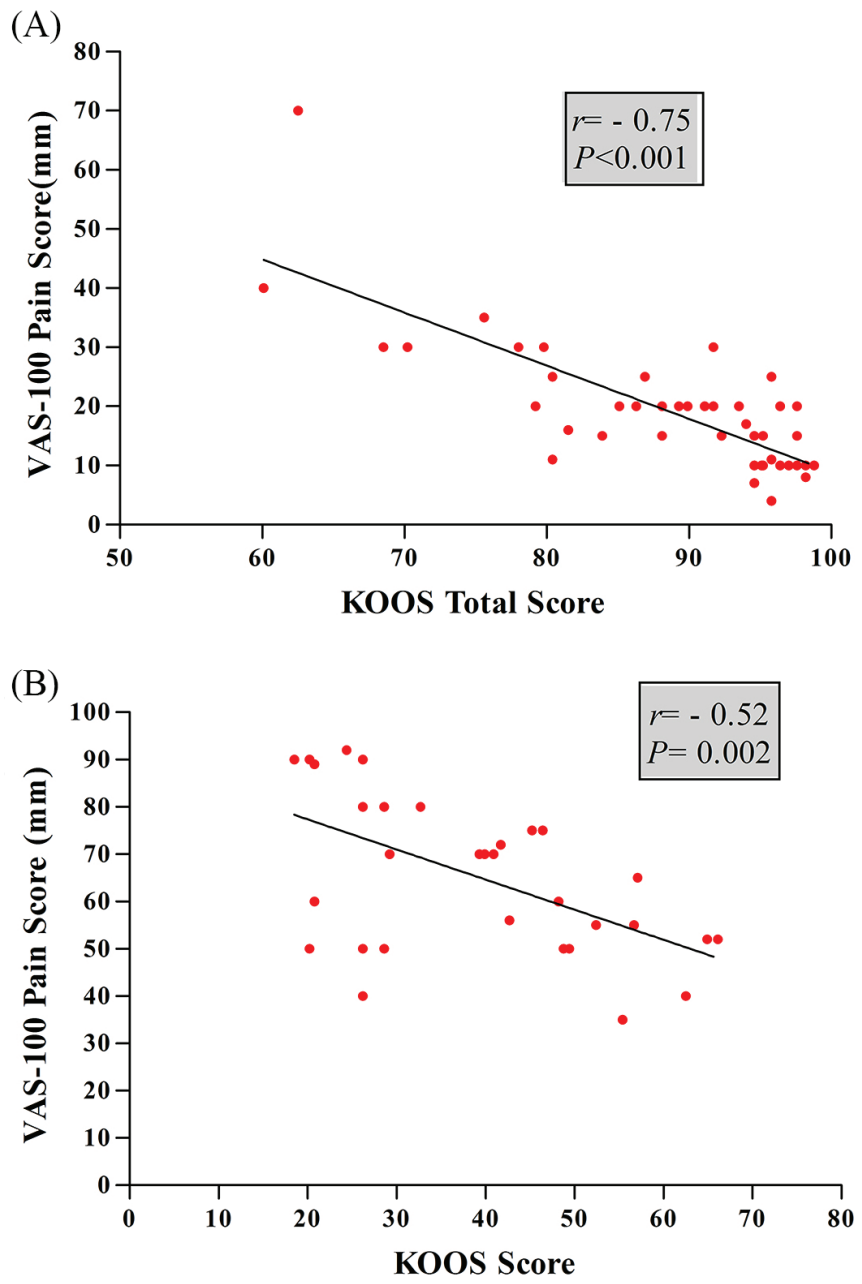

FIGURE 3 - Spearman's correlation between KOOS and VAS100 scores in both treatment groups after 90 days. $r$ : Spearman's correlation coefficient; A: Mlx+Res group; $\mathrm{B}$ : Mlx+placebo group.

significant correlation was observed in both groups. Moreover, evaluation of the sensitivity and specificity of the two methods using the ROC curve demonstrated a highly significant $(P<0.0001)$ overlap for AUC in both treatment groups (AUC=1.0, Figure 4). 


\section{ROC of KOOS vs WOMAC}

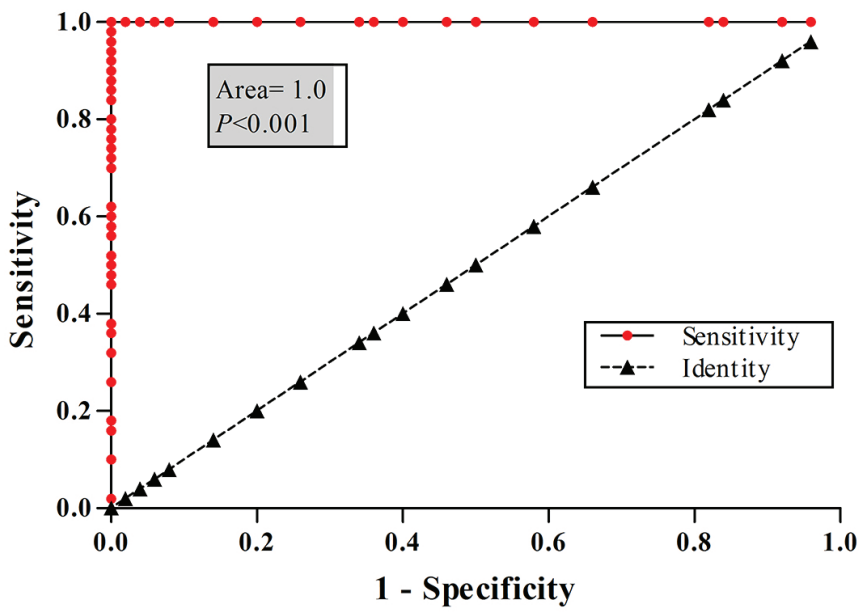

FIGURE 4 - ROC curve illustrating the sensitivity and specificity for different values of KOOS corresponding to a WOMAC. ROC: Receiver operating characteristic curve.

\section{DISCUSION}

Despite advances in understanding the mechanisms and treatment of OA-associated pain, many OA patients experienced various levels of acute and chronic pain, which impairs their daily living activities and quality of life (Wang et al., 2016). Moreover, untreated OA pain may lead to serious negative impacts, like those reported in the baseline clinical scores of the included patients (Table I). The present study is the first randomised controlled clinical trial to provide clinical evidence regarding the efficacy of resveratrol in improving the symptoms and pain associated with knee OA, which can provide the basis for the clinical implementation of oral resveratrol in knee osteoarthritis pain. Since the symptoms of osteoarthritis were assessed mainly by pain, the outcomes of the present study were quantitatively evaluated by the severity of pain. Commonly, most studies in this regard used VAS of pain or pain index in WOMAC, while others utilised different techniques, including frequencies of painkiller use during the study or qualitatively identifying pain descriptors (Nakagawa et al., 2014; Cedraschi et al., 2013). Although Knee OA is a chronic musculoskeletal disorder that is not life-threatening, it may seriously influence the emotional, daily physical and social activity of patients. Accordingly, it negatively impacts the health-related quality of life. Meanwhile, effective pharmacological treatment not only improves pain and mobility but also the quality of life as a whole. Due to high prevalence of OA, its association with high costs of healthcare induced by the consumption of drugs and loss of work force and productivity is highly recognised (Gupta et al., 2005). Therefore, increasing interest in pharmacological agents that modulate OA progression and limit the need for surgical interventions were strongly encouraged. To the best of our knowledge, the present study is the first clinical trial on the effects of resveratrol, as a herbal supplement, on the pain and associated dysfunction scores in patients with evidence of knee OA. Using the KOOS, WOMAC and VAS questionnaire instruments approach, resveratrol supplementation results in significant decreases of total knee pain scores, and an improved disability index and overall health scores compared with placebo. These results support a role for naturally-derived supplements like resveratrol as an alternative or complementary treatment option in pain management that may also reduce surrogate markers of disease progression in knee OA. While our results show that resveratrol is superior to placebo, we noticed clinically important alterations in self-rated outcomes in both treatment groups. These were most evident with pain score; however, we also observed such changes in the KOOS and WOMAC symptom score. In the present study, 90 days of resveratrol use resulted in a significant improvement in the clinical scores of OA pain and disability in participants with knee OA. These clinical observations are in line with the available data showing that resveratrol reduces pain, inflammation, and oedema, as well as articular destruction in experimental models of arthritis (Wang, Chen, Wang, 2016; Wang et al., 2017). Together with the previously reported data, the present finding supports the analgesic activity of dietary polyphenols in patients with mild-tomoderate knee pain, and suggests that it is in tune with the results of many pilot studies reported regarding the adjuvant use of polyphenols and other supplements during the treatment of inflammatory musculoskeletal disorders like knee OA and rheumatoid arthritis (Hussain et al., 2009; Hussain et al., 2016). Although the exact mechanism behind the impact of resveratrol on improving OA symptoms in a clinical setting has not been well described, a series of animal experiments conducted in the past few years has demonstrated that the oral administration of resveratrol is capable of significantly preventing the progression of osteoarthritis (Gu et al., 2016), and also exhibits a potent analgesic effect in different animal models (Wang et al., 2017; Bazzo et al., 2013). Despite the large output of preclinical studies dealing with the analgesic and antiinflammatory activities of resveratrol, there is a relative lack of clinical trials investigating the analgesic effect of resveratrol (Nguyen et al., 2017; Vang et al., 2011). Many updated regimens for managing $\mathrm{OA}$ focus on relieving pain and stiffness and improving physical function as important goals of therapy (Suokas et al., 2014; Moyer et al., 2014). However, the aetiology and progression of OA have been understood to have an inflammatory basis during the early 
stages of disease (Felson, 2006), which might be associated with tissue damage, especially the destruction of cartilage. In OA, many important mediators of the altered metabolism and increased catabolism of joint tissues are formed based on the cartilage damage and increased circulating inflammatory factors, including pro-inflammatory cytokines (Kapoor et al., 2011). Moreover, inflammation and pain are firmly related to human health, and there is always strong interplay between pain and inflammation. Thus, inflammation is regarded as an important part of the pathogenesis of OA and is strongly correlated with the deterioration of subscales targeted through KOOS and WOMAC. In tune with previous reports, our findings support the analgesic effects of dietary polyphenols in adults with mild-to-moderate knee pain. The assessment of OA pain has been largely determined by questionnaires such as those based on quality of life indicators. Meanwhile, radiographic imaging and physical examination have been used to stage the disease. We used KOOS, WOMAC and VAS questionnaires, which have been widely employed to assess knee pain, quality of life, and disability in patients with knee OA (Mahler et al., 2016). Based on the present findings, resveratrol consistently improved pain scores, as observed across all sub-scales of KOOS and WOMAC, evaluating constant, intermittent, and total pain; they also lowered the associated scores, reflecting functional improvement. We observed a difference in pain scores assessed by VAS in our participants, which could be explained by the visual expression of general pain intensity used in VAS scoring compared to the knee OA-specific magnitude of knee pain numerically rated by the KOOS and WOMAC questionnaires. Another notable finding of the present study is the high level of agreement between clinical outcomes when assessed by either KOOS or WOMAC in Iraqi patients with knee OA (Figure 4), which was in line with previously reported data indicating that KOOS is an instrument with improved validity that may be at least as responsive as WOMAC (Roos, Toksvig-Larsen, 2003). Based on the limitations and strengths of each assessment tool for pain, it is practically recommended to utilise more than one instrument to capture the multi-dimensional aspects of OA pain. The present study has limitations that affect the interpretation of findings. These include the small sample size, and the absence of a dose-response design to assess effects at low vs. high doses of resveratrol. The included patients had mild-to-moderate radiographic knee OA at baseline (Kellgren-Lawrence scores of 1-3) and mild-to-moderate knee pain. Whether patients with more severe knee OA (Kellgren-Lawrence score of 4) and higher levels of pain would benefit from resveratrol-based treatment needs further investigation. Moreover, we did not evaluate other biomarkers related to oxidative damage, or simultaneously measure these biomarkers in synovial fluid that would provide a more accurate determination of changes specific to the knee. Also, because of the relatively short follow up duration, we did not assess the radiological outcomes at the end of the trial. Meanwhile, the strengths of the present study include a randomised, multicentre, double blinded placebo controlled study design, which consider most of the inter-individual variations in parallel arm studies. Additionally, we excluded patients who were taking any kind of supplements, especially those containing polyphenols, or other herbal supplements for pain relief, and were therefore able to exclude potential confounding by these factors.

\section{CONCLUSIONS}

This pilot study demonstrates evidence of the role of resveratrol, when used in combination with meloxicam, in improving pain in patients with mild-to-moderate knee OA compared with placebo. It also suggests that intervention with a dietary supplement, i.e. the addition of resveratrol, may have a significant impact on pain and overall quality of life in patients with knee OA.

\section{ACKNOWLEDGEMENTS}

The data were abstracted from a PhD thesis submitted by Bushra H. Marouf to the College of Medicine, University of Sulaimani. The project was totally supported by the University of Sulaimani (Certificate 42 in $21 / 11 / 2016$ ). The authors gratefully thank the kind supports from Shar Teaching Hospital and the Specialized Rheumatology Center in Sulaimani City.

\section{CONFLICTS OF INTEREST}

The authors declare no conflict of interest.

\section{REFERENCES}

Altman R, Asch E, Bloch D, Bole G, Borenstein D, Brandt K, et al. Development of criteria for the classification and reporting of osteoarthritis. Classification of osteoarthritis of the knee. Diagnosis and therapeutic criteria committee of the American rheumatism association. Arthritis Rheum. 1986;29(8):1039-1049.

Bazzo KO, Souto AA, Lopes TG, Zanin RF, Gomez MV, Souza $\mathrm{AH}$, et al. Evidence for the analgesic activity of resveratrol in acute models of nociception in mice. J Nat Prod. 2013;76(1):13-21. 
Bellamy N, Buchanan WW, Goldsmith CH, Campbell J, Stitt LW. Validation study of WOMAC: a health status instrument for measuring clinically important patient relevant outcomes to anti-rheumatic drug therapy in patients with osteoarthritis of the hip or knee. J Rheumatol. 1988;15(12):1833-1840.

Brown VA, Patel KR, Viskaduraki M, Crowell JA, Perloff M, Booth TD, et al. Repeat dose study of the cancer chemopreventive agent resveratrol in healthy volunteers: safety, pharmacokinetics, and effect on the insulin-like growth factor axis. Cancer Res. 2010;70(22):9003-9011.

Buckwalter JA, Martin JA. Sports and osteoarthritis. Curr Opin Rheumatol. 2004;16(5):634-639.

Cedraschi C, Delézay S, Marty M, Berenbaum F, Bouhassira D, Henrotin Y, et al. Let's talk about OA Pain: A qualitative analysis of the perceptions of people suffering from OA. Towards the development of a specific pain OA-related Questionnaire, the Osteoarthritis Symptom Inventory Scale (OASIS). PLoS One. 2013;8(11):e79988.

Felson DT. Clinical practice. Osteoarthritis of the knee. N Engl J Med. 2006;354(8):841-848.

Gu H, Li K, Li X, Yu X, Wang W, Ding L, et al. Oral resveratrol prevents osteoarthritis progression in C57BL/6 J mice fed a high-fat diet. Nutrients. 2016;8(4):233.

Gupta S, Hawker GA, Laporte A, Croxford R, Coyte PC. The economic burden of disabling hip and knee osteoarthritis (OA) from the perspective of individuals living with this condition. Rheumatology (Oxford). 2005;44(12):1531-1537.

Haq SA, Davatchi F. Osteoarthritis of the knees in the COPCORD world. Int J Rheum Dis. 2011;14(2):122-129.

Hayami T. Osteoarthritis of the knee joint as a cause of musculoskeletal ambulation disability symptom complex (MADS). Clin Calcium. 2008;18(11):1574-1580.

Hussain SA, Jassim NA, Numan IT, Al-Khalifa II, Abdullah TA. Anti-inflammatory activity of silymarin in patients with knee osteoarthritis: A comparative study with piroxicam and meloxicam. Saudi Med J. 2009;30(1):179-184.

Hussain SA, Mortada AH, Jasim NA, Gorial FI. Silibinin improves the effects of methotrexate in patients with active rheumatoid arthritis: Pilot clinical study. Oman Med J. 2016;31(4):263-269.
Jiang M, Li X, Yu X, Liu X, Xu X, He J, et al. Oral administration of resveratrol alleviates osteoarthritis pathology in C57BL/6J mice model induced by a high-fat diet. Mediators Inflamm. 2017;2017:7659023.

Johnson VL, Hunter DJ. The epidemiology of osteoarthritis. Best Pract Res Clin. Rheumatol. 2014;28(1):5-15.

Johnson WD, Morrissey RL, Usborne AL, Kapetanovic I, Crowell JA, Muzzio M, et al. Sub-chronic oral toxicity and cardiovascular safety pharmacology studies of resveratrol, a naturally occurring polyphenol with cancer preventive activity. Food Chem Toxicol. 2011;49(12):3319-3327.

Kapoor M, Martel-Pelletier J, Lajeunesse D, Pelletier JP, Fahmi $\mathrm{H}$. Role of pro-inflammatory cytokines in the pathophysiology of osteoarthritis. Nat Rev Rheumatol. 2011;7(1):33-42.

Kellgren JH, Lawrence JS. Radiological assessment of osteoarthrosis. Ann Rheum Dis. 1957;16(4):494-502.

Kraus VB, Blanco FJ, Englund M, Karsdal MA, Lohmander LS. Call for standardised definitions of osteoarthritis and risk stratification for clinical trials and clinical use. Osteoarthritis Cartilage. 2015;23(8):1233-1241.

La Porte C, Voduc N, Zhang G, Seguin I, Tardiff D, Singhal N, et al. Steady-state pharmacokinetics and tolerability of transresveratrol $2000 \mathrm{mg}$ twice daily with food, quercetin and alcohol (ethanol) in healthy human subjects. Clin Pharmacokinet. 2010;49(7):449-454.

Loeser RF, Goldring SR, Scanzello CR, Goldring MB. Osteoarthritis: a disease of the joint as an organ. Arthritis Rheum. 2012;64(4):1697-1707.

Ma C, Wang Y, Dong L, Li M, Cai W. Anti-inflammatory effect of resveratrol through the suppression of NF- $\kappa$ B and JAK/STAT signalling pathways. Acta Biochim Biophys Sin. (Shanghai) 2015;47(3):207-213.

Mahler E, Cuperus N, Bijlsma J, Vliet Vlieland T, van den Hoogen F, den Broeder AA, et al. Responsiveness of four patientreported outcome measures to assess physical function in patients with knee osteoarthritis. Scand J Rheumatol. 2016;45(6):518527.

Mobasheri A, Batt M. An update on the pathophysiology of osteoarthritis. Ann Phys Rehabil Med. 2016;59(5):333-339. 
MobasheriA, Bay-JensenAC, van Spil WE, Larkin J, Levesque MC. Osteoarthritis Year in Review 2016: biomarkers (biochemical markers). Osteoarthritis Cartilage. 2017;25(2):199-208.

Moura Mdel G, Lopes LC, Biavatti MW, Busse JW, Wang L, Kennedy SA, et al. Brazilian oral herbal medication for osteoarthritis: A systematic review protocol. Syst Rev. 2016;5:86.

Moyer RF, Ratneswaran A, Beier F, Birmingham TB. Osteoarthritis year in review 2014: Mechanics - Basic and clinical studies in osteoarthritis. Osteoarthritis Cartilage. 2014;22(12):1989-2002.

Nakagawa Y, Mukai S, Yamada S, Matsuoka M, Tarumi E, Hashimoto T, et al. Short-term effects of highly-bioavailable curcumin for treating knee osteoarthritis: A randomised, doubleblind, placebo-controlled prospective study. J Orthop Sci. 2014;19(6):933-939.

Nelson AE, Allen KD, Golightly YM, Goode AP, Jordan JM. A systematic review of recommendations and guidelines for the management of osteoarthritis: the chronic osteoarthritis management initiative of the U.S. bone and joint initiative. Semin Arthritis Rheum. 2014;43(6):701-712.

Neves AR, Nunes C, Reis S. Resveratrol induces ordered domains formation in biomembranes: Implication for its pleiotropic action. Biochim Biophys Acta. 2016;1858(1):12-18.

Nguyen C, Savouret JF, Widerak M, Corvol MT, Rannou F. Resveratrol, potential therapeutic interest in joint disorders: A critical narrative review. Nutrients. 2017;9(1):45.

Patel KR, Scott E, Brown VA, Gescher AJ, Steward WP, Brown K. Clinical trials of resveratrol. Ann N Y Acad Sci. 2011;1215:161-169.

Roos EM, Toksvig-Larsen S. Knee injury and Osteoarthritis Outcome Score (KOOS)-validation and comparison to the WOMAC in total knee replacement. Health Qual Life Outcomes. 2003;1:17.

Salavati M, Akhbari B, Mohammadi F, Mazaheri M, Khorrami M. Knee injury and Osteoarthritis Outcome Score (KOOS); reliability and validity in competitive athletes after anterior cruciate ligament reconstruction. Osteoarthritis Cartilage. 2011;19(4):406-410.
Suokas AK, Sagar DR, Mapp PI, Chapman V, Walsh DA. Design, study quality and evidence of analgesic efficacy in studies of drugs in models of OA pain: A systematic review and a meta-analysis. Osteoarthritis Cartilage. 2014;22(9):1207-1223.

Tomé-Carneiro J, Larrosa M, Yáñez-Gascón MJ, Dávalos A, GilZamorano J, Gonzálvez M, et al. One-year supplementation with a grape extract containing resveratrol modulates inflammatoryrelated microRNAs and cytokines expression in peripheral blood mononuclear cells of type 2 diabetes and hypertensive patients with coronary artery disease. Pharmacol Res. 2013;72:69-82.

Vang O, Ahmad N, Baile CA, Baur JA, Brown K, Csiszar A, et al. What is new for an old molecule? Systematic review and recommendations on the use of resveratrol. PLoS One. 2011;6(6):e19881.

Varas-Lorenzo C, Riera-Guardia N, Calingaert B, Castellsague J, Salvo F, Nicotra F, et al. Myocardial infarction and individual non-steroidal anti-inflammatory drugs meta-analysis of observational studies. Pharmacoepidemiol Drug Saf. 2013;22(6):559-570.

Wang G, Hu Z, Song X, Cui Q, Fu Q, Jia R, et al. Analgesic and anti-inflammatory activities of resveratrol through classic models in mice and rats. Evid Based Complement Alternat Med. 2017;2017:5197567.

Wang P, Yang L, Liu C, Wei X, Yang X, Zhou Y, et al. Effects of whole body vibration exercise associated with quadriceps resistance exercise on functioning and quality of life in patients with knee osteoarthritis: a randomised controlled trial. Clin Rehabil. 2016;30(11):1074-1087.

Wang ZM, Chen YC, Wang DP. Resveratrol, a natural antioxidant, protects monosodium iodoacetate-induced osteoarthritic pain in rats. Biomed Pharmacother. 2016;83:763-770.

Williams LD, Burdock GA, Edwards JA, Beck M, Bausch J. Safety studies conducted on high-purity trans-resveratrol in experimental animals. Food Chem Toxicol. 2009;47(9):2170-2182.

Zhang W, Moskowitz RW, Nuki G, Abramson S, Altman $\mathrm{RD}$, Arden N, et al. OARSI recommendations for the management of hip and knee osteoarthritis, part I: critical appraisal of existing treatment guidelines and the systematic review of current research evidence. Osteoarthritis Cartilage. 2007;15(9):981-1000.

Received for publication on $27^{\text {th }}$ November 2017 Accepted for publication on $23^{\text {rd }}$ March 2018 